(C) $\{$ Owner/Author $\mid \mathrm{ACM}\}\{2016\}$. This is the author's version of the work. It is posted here for your personal use. Not for redistribution. The definitive Version of Record was published in ACM Digital Library, https://doi.org/10.1145/3011141.3011489 


\section{SEMANTICALLY-ENHANCED ADVERTISEMENT RECOMMENDER SYSTEMS IN SOCIAL NETWORKS}

\author{
Ali Pazahr \\ Department of Computer Science \\ Islamic Azad University \\ Ahvaz, Iran \\ +989166157215 \\ Pazahr@iauahvaz.ac.ir
}

\author{
Dr. J. Javier Samper Zapater \\ IRTIC \\ University of Valencia \\ Valencia, Spain \\ +34963543567 \\ jsamper@irtic.uv.es
}

\author{
Dr. Francisco García Sánchez \\ Department of Informatics and Systems \\ University of Murcia \\ Murcia, Spain \\ +3486888 8107 \\ frgarcia@um.es
}

\author{
Dr. Carmen Botella \\ IRTIC \\ University of Valencia \\ Valencia, Spain \\ +34963543424 \\ Carmen.botella@uv.es
}

\author{
Dr. Rafael Martinez \\ IRTIC \\ University of Valencia \\ Valencia, Spain \\ +34963543564 \\ Rafael.Martinez@uv.es
}

\begin{abstract}
Providing recommendations on social systems has been in the spotlight of both academics and industry for some time already. Social network giants like Facebook, LinkedIn, Myspace, etc., are eager to find the silver bullet of recommendation. These applications permit clients to shape a few certain social networks through their day-by-day social cooperative communications. In the meantime, today's online experience depends progressively on social association. One of the main concerns in social network is establishing a successful business plan to make more profit from the social network. Doing a business on every platform needs a good business plan with some important solutions such as advertise the products or services of other companies which would be a kind of marketing for those external businesses. In this study a philosophy of a system speaking to of a comprehensive structure of advertisement recommender system for social networks will be presented. The framework uses a semantic logic to provide the recommended products and this capability can differentiate the recommender part of the framework from classical recommender methods. Briefly, the framework proposed in this study has been designed in a form that can generate advertisement recommendations in a simplified and effective way for social network users.
\end{abstract}

\section{CCS Concepts}

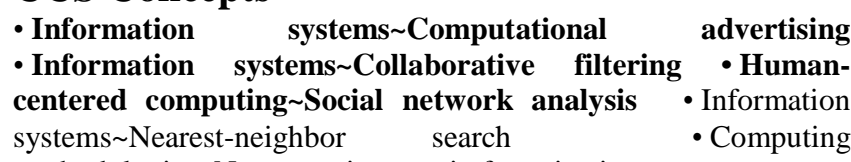

methodologies Non-negative matrix factorization

\section{Keywords}

social network; comprehensive structure; recommender systems; advertisement; semantic technologies

\section{INTRODUCTION}

Promoting on the social communities is a multi-billion dollar market and has turned great income into the popular social networks for their business (1). To get ready advertisements and convey them to conceivably intrigued clients, these social media platforms take in a divination model for all users according to the users' interests and activities. One of the specifications of old systems of social network was that they were sending all advertisement for all of users without considering their preferences (2) (3) (4) (5) like what was happening to email marketing. Another characteristic of old social networks was that the procedure of choosing, preparing and sending of advertisements were manual while nowadays for the new level of facilities in social networks with new related technologies, it is not possible to do it in the previous traditional form. So it is important to select a solution to generate suggestion of the products for the users based on an automatic mechanism.

Notwithstanding, as client interests frequently advance gradually, the client may wind up accepting tedious promotions. So it is essential for the organizations of social networks to upgrade the logic of their framework's software to provide qualified advertisement based on the users' feedback. The ability of knowledge contribution nowadays is unmatched ever. At no other time have such a large 
number of inventive and proficient individuals been associated by such a productive, all-inclusive system. The expenses of social occasion and registering over their commitments have come down to the point where new organizations with extremely humble spending plans give imaginative new administrations to a great number of online members. Collective intelligence is an amazing insight that can have numerous constructive outcomes on social networks. The outcome nowadays is remarkable broadness of data and variety of point of view, and a society of mass investment that supports a wellspring of freely accessible substance (6).

As opposed to the tremendous number of things in recommender frameworks, every client regularly just rates a couple. In this way, the user/thing rating grid is commonly extremely scanty. It is troublesome for recommender frameworks to precisely quantify client likenesses from those predetermined number of audits. A related issue is the Cold-start issue (7). Notwithstanding for a framework that is not especially meagre, when a client at first joins, the framework has none or maybe just a couple audits from this client. In this manner, the framework cannot precisely translate this current client's inclination. The combination of social networks can hypothetically enhance the execution of current recommender frameworks. To start with, as far as the forecast precision, the extra data about clients and their companions acquired from social networks enhances the comprehension of client practices and appraisals.

The main objective of this paper is to study and design of a framework for advertisement recommender system in social networks that can be enriched by semantic technologies. Although there are many applications and solutions about recommender systems, in this work the focus is on designing a robust framework with a suitable performance which can be implemented on every social network to extend their business purposes. In order to achieve this goal, a reduced number of more specific tasks have been identified: (1) overcoming the primary constraints of stateof-the-art methods, (2) improving the quality of recommendations and the performance of the recommender system, (3) providing a proof-of-concept implementation by setting up the suggested framework on a real software platform, (4) boosting portability and reliability, and (5) keeping an acceptable level of security for the framework.

The rest of the paper is organized as follows. In Section 2 current advertisement recommender systems are reviewed. The methodology proposed in this work is described in Section 3. In Section 4 an extensive evaluation of the methodology is provided. Conclusions and future work are put forward in Section 5.

\section{Related Works}

Research on recommender systems has been very active during the last few years. In (8) Zadeh and Moshkenani expressed that with having an advertisement according to user favorites and interests was targeted by mining his/her interactions in digital social networks semantically. In fact, by finding people that have more chance to accept the desired advertisement, system has a more success over the traditional method at a lowered cost.

In (9) a recommendation system was proposed which utilized semantic web technology and healthcare social networking to provide personalized recommendation to speed patient recovery and improve healthcare outcomes. Extensive experiments have been performed to evaluate the performance of the system. The results demonstrated the effectiveness of the proposed strategy.

In (10) the authors propose a recommender system exploiting the possibilities that Web 2.0 offers, and goes one step further to increase coupon redemptions, by utilizing social networks as tools to add extra information to the system and reach new consumers.

The work presented in (11) was introduced as a system that was providing users with a service for recommending categories by utilizing social folksonomy with clustered data. Further, a method to reduce the dimension of vectors by removing meaningless words in the contents is introduced. A recommendation approach based on quantified social tie strength was described in (12). The authors proposed an unsupervised method to estimate tie strength from user similarity and online social interactions. Then the approach improves the social recommendation with quantified social tie strength. Experiments are made on a large book rating dataset from Douban.com. The experimental results show that this approach can effectively improve the recommendation accuracy.

In (13) a research model was tested in an online experiment using Facebook data and it was developed for the use case of online news with 193 participants. The structural equation model results show that a strong tie relationship has positive influence on the value of a recommendation. The credibility of the recommending person and the recommendation's media source affect the value of a recommendation as well.

In (14) a new group recommendation method was demonstrated, which combined topic identification and social networks for group recommendation. In detail, they firstly identified different topical sub-groups by topics in social networks. Secondly, different user factors were used to calculate the user influence (including individual and social) on the topical sub-groups, which can depict the topical subgroup characteristics in different points of view. Experimental results demonstrate that the proposed method can improve the prediction accuracy of the group recommendation.

By reviewing of related works, it is possible to see that most of similar frameworks have designed based on the composition of the standard techniques. The main motivation for doing this research was that there were several similar related works in which there were some problems and limitations whether on base of design or operation and it was a good start point to analyze the similar works, check their functionality and propose a more 
comprehensive framework which can overcome those problems and limitations. For example, one of the most obvious problems that the users of social networks face is that the results of the recommendation engine of those systems were not very interesting for the users or even some times the recommended products or services were irrelevant. Furthermore, the other problem was the lack of existence of a feedback consideration to enrich the quality of the recommendations.

This motivation could help to design and develop a novel framework for advertisement recommender systems in social networks. Moreover, the current research can demonstrate a suitable solution for the available systems and enhance them to operate better than before. The novelties that are used in this study's framework can make evolutions in the future recommender system, which work on the social media platform.

\section{PROPOSED METHOD}

Based on the users' interests, the recommendation engine uses a hybrid recommendation method to find the products that can be best suited to users' preferences. In order to define the values of the rates matrix, five levels of interests to the products are identified. For each level of the users' activity a number is assigned and each level of interest is determined by the users' activity. The variable "irank" (interest rank) is assigned for the user interest level. If the product is searched by the user, "irank" is set to 5 . In this research, searching can be used as a level of interest. If the product is shown and browsed for the user along with its details, "irank" is set to 4 . It means that the user pays more attention to the product rather than just searching. Next, if the user interested in the product, there is a possibility to rate the product from 1 to 3 which can set "irank" from 3 to 1 respectively.

Another key variable in the proposed methodology is "trank" (product rank), which refers to the rank of the advertised product in social network related to a specific producer. This rank is populated from users' activities on social networks and indicates the popularity of the product among users of social network. Two linear formulas, one considering demographic issues (Equation 1) and another context-aware issues (Equation 2), are used to calculate the final rate, namely "itrate", for each product, which is qualified to be recommended to the current user (15).

$$
\begin{gathered}
\text { itrate }=\operatorname{arrSimilarUsersDemo}[\mathrm{i}, 1] /(5 * \mathrm{irank} * \text { trank }) \\
\begin{array}{r}
\text { Equation } 1 \text { Calculation of itrate for the Demographic part of } \\
\text { recommendations }
\end{array} \\
\text { itrate = arrSimilarUsersCtx }[\mathrm{i}, 1] /(6 * \mathrm{irank} * \text { trank }) \\
\text { Equation } 2 \text { Calculation of itrate for the Context-aware part of } \\
\text { recommendations }
\end{gathered}
$$

The value of itrate operates as a score for the product to show the strength of interest for the user. It is calculated using irank, trank, users' similarity, and a fixed ratio depending to the recommendation techniques which is 5 for demographic and 6 for context-aware recommender method. The labels "demo" and "ctx" are assigned to recommendations by demographic and context-aware methods and this information along with the username of the current user are added to the table as "Recs". To prevent saving repetitive recommendation in these two methods, before entering the recommendation to database, all of previous recommendations which have been made before by these two methods, are replaced by the new recommendations.

Up to now, the engine has provided a list of products to be recommended as advertisements by two techniques. But there are still two recommendation techniques remained to be considered.

\subsection{Steps of Recommendation}

For the first part of the recommendations with contentbased filtering, a list of the current user's interesting products whose irate from table "Interests" is between 1 and 3 was prepared. The tags brought from social network and were set for the products in this list, were extracted. Thus these tags which were related to the interesting products for the current user were found. It means that the current user prefers the products containing these tags. Then the tags were sorted in descending order based on their frequency in the recent list. So the more tags preceded, the more current user interested in for each tag, three products from the table "products" for which the user has not visited and the products which have the most "trank" value earned from social network and contain the tag, were selected.

For the second part of the recommendations with contentbased filtering, the music tracks which were rated by the current user were considered. Then, the top three of the most popular other music tracks distinguished by "rank" of tracks with the same artist were found.

The product details along with the label "cb" indicating content-based recommended products, username of current user and "itrate" which was calculated using Equation 3 were added to the table "Recs":

$$
\text { itrate }=1 /(6 * \text { trank })
$$

\section{Equation 3 Calculation of itrate for Content-based Filtering part of recommendations}

For the collaborative filtering recommendations, the method of $\mathrm{k}$ nearest neighborhood $(\mathrm{kNN})$ was used to find the most similar users. First, registered users along with their interesting products were considered. This information was used from the table "Interests". Accordingly, the matrix "rates" as rates [userid, productid] was defined and the rate values given to the products by the users were entered to this matrix based on users' interests or their activities. The reason for choosing the table "Interests" as the dataset for filling matrix rates' values is that the rates computable by recommendation engine, could not be 
calculated by the products for which no user had rated before. Therefore, the products without any rate value by any user were not taken into consideration (16). The set of items rated by each both users in the matrix rates should be considered. The matrix of " $w$ " containing all weights or the amounts of closeness of users together as $\mathrm{w}_{\mathrm{a}, \mathrm{u}}$ was prepared according to the Equation 4:

$$
w_{a, u}=\frac{\sum_{i \in I}\left(r_{a, i}-\bar{r}_{a}\right)\left(r_{u, i}-\bar{r}_{u}\right)}{\sqrt{\sum_{i \in I}\left(r_{a, i}-\bar{r}_{a}\right)^{2} \sum_{i \in I}\left(r_{u, i}-\bar{r}_{u}\right)^{2}}}
$$

Equation 4 Calculation of $w_{a, u}$

Where $\mathrm{I}$ is the set of products rated by each both users, $r_{u, i}$ is the rating given to product $i$ by user $u$, and $\overline{\mathrm{r}}_{\mathrm{a}}$ is the mean rating given by user $u$. Each of unrated elements of the matrix "w" was calculated by the Equation 5:

$$
p_{a, i}=\bar{r}_{a}+\frac{\sum_{u \in K}\left(r_{u, i}-\bar{r}_{u}\right) \times w_{a, u}}{\sum_{u \in K} w_{a, u}}
$$

Equation 5 Calculation of $p_{a, i}$

Where $\mathrm{p}_{\mathrm{a}, \mathrm{i}}$ is the prediction for the active user a for product $\mathrm{i}, \mathrm{w}_{\mathrm{a}, \mathrm{u}}$ is the similarity between users a and $\mathrm{u}$, and $\mathrm{K}$ is the neighborhood or set of most similar users. If the collaborative filtering recommendations had been previously saved to the table "Recs", they were replaced by these up-to-date recommended products. For each user, the highest element values, which were calculated by the last equation, were added to the table "Recs" along with the other details containing username, product id, the label "cf" indicating the recommendation technique and "itrate" which was computed by the Equation 6:

$$
\text { Itrate }=\text { rates }[\text { useridindex, productidindex }] / 10
$$

\section{Equation 6 Calculation of itrate for Collaborative Filtering part of recommendations}

Finally, among total prepared recommendations through four techniques from the table "Recs", a list of top ten products with highest "itrate" values, is shown to the current user.

One interesting novelty in this recommendation engine is that a group of calculated recommended products for the current user which even could be found repeatedly but estimated by different techniques with different "itrate" values, are aggregated based on "itrate" values. For this purpose, a SQL operation of "Group by" userid and productid is done along with considering a total "itrate" of recommendations using four techniques as Total_itrate. As a result, the earned records of the recent dataset potentially show the probable interest rates of the user about the products. Finally, these records should be sorted by Total_itrate in order to find the best recommendations. In other words, the method of recommendation is ranking-

\begin{tabular}{|c|c|c|c|c|c|c|}
\hline userid & productid & itrate & $\mathrm{rx}$ & userid & productid & Total_itrate \\
\hline 1754624498 & 975 & 0.167 & $\mathrm{cb}$ & 1754624498 & 975 & 0.167 \\
\hline 1754624498 & 976 & 0.083 & $c b$ & 1754024498 & 976 & 0.083 \\
\hline 1754624498 & 977 & 0.056 & $c b$ & 1754624498 & 977 & 0.056 \\
\hline 1754624498 & 10599 & 0.158 & cf & 1754624498 & 10599 & 0.159 \\
\hline 1754624498 & 10599 & 0.001 & $\mathrm{ctx}$ & 1754624498 & 85637 & 0.167 \\
\hline 1754624498 & 85637 & 0.167 & $c b$ & 1754624498 & 85638 & 0.083 \\
\hline 1754624498 & 85638 & 0.083 & $c b$ & 1754624498 & 85639 & 0.056 \\
\hline 1754624498 & 85639 & 0.056 & $c b$ & 1754624498 & 108351 & 0.167 \\
\hline 1754624498 & 108351 & 0.167 & $c b$ & 1754624498 & 108352 & 0.083 \\
\hline 1754624498 & 108352 & 0.083 & $\mathrm{cb}$ & 1754624498 & 108353 & 0.056 \\
\hline 1754624498 & 108353 & 0.056 & $\mathrm{cb}$ & 1754624498 & $\mathbf{A}_{125314}$ & 0.331 \\
\hline 1754624498 & 125314 & 0.133 & the & 1754624498 & 125329 & 0.053 \\
\hline 1754624498 & 125314 & 0.158 & cf & & & \\
\hline 1754624498 & 125314 & 0.040 & demo & & & \\
\hline 1754624498 & 125329 & 0.012 & demo & & & \\
\hline 1754624498 & 125329 & 0.041 & $\mathrm{ctx}$ & & & \\
\hline
\end{tabular}
based (Figure 1). The top most records of the recent dataset will be shown to the user as the recommendations. 
So for each distinct product, its "itrate" is calculated using Equation 7:

$$
\begin{aligned}
& \text { Total_itrate }=\sum_{i=1}^{n}(\text { itrate }(i)) \\
& \text { Equation } 7 \text { Calculation of Total_itrate }
\end{aligned}
$$

Where " $n$ " at most can be 4 , equal to the four used techniques and depends on count of calculated recommendations and "i" is referring to the number of each used technique.

Through recommended products interface, the users have the choice to make decision about the recommended products and rate them based on how far they are matched to the users' taste. With this feature, it is possible to arrange an evaluation for the framework.

The greater part of recommendation frameworks is utilizing one or blend of two procedures as a half and half system, while the recommended structure in this exploration uses the advantages of four methods. Besides, some specialized oddities completely enabled this system.

\subsection{Segments of the Structure}

In this section, the rationale of the structure will be presented and clarified what segments it has and how they function. Every part of the structure was shown in points of interest furthermore the relations between the segments have been spoken to. Moreover, a few key variables which are utilized as a part of the system have been presented alongside their usefulness.

For the collaborative filtering recommendations, the technique for $\mathrm{k}$ closest neighborhood ( $\mathrm{kNN})$ was utilized to locate the most comparative clients. Initially, enrolled clients alongside their intriguing items were considered. This data was utilized from the table "Interests". Likewise, the matrix rates [userid, productid] was characterized and the rate values given to the items by the users were entered to this framework taking into account users' interests or their exercises. The arrangement of items evaluated by each both users in the framework rates ought to be considered. The displayed structure is by and large so that can be connected for every informal organization working for even other media like video or picture. In any case, for delineating the system and make it all the clearer, it is ideal to practice and execute it over an informal organization to watch how the strategy functions in a genuine stage and how is the nature of the structure for evaluating its execution. The social network Last.fm is chosen for this area as an appropriate contextual analysis.

A web application for performing the structure was produced. As to the subject of the paper, Semanticallyenhanced Advertisement Recommender Systems In Social networks, the web application was named as SARSIS which is accessible on the web. For this situation the web application was produced in MS Visual Studio .NET and with the innovation ASP.NET.

For testing the web application, the needed information for working is provided so that it is possible to work with the system and watch how it works in practical.

The dataset that was utilized as a part of the exploratory part of the proposition was displayed. Since no appropriate dataset coordinated to the structure, could be discovered, it was ideal to gather genuine information from the interpersonal organization straightforwardly. The DBMS MS SQL Server was utilized for sparing the dataset. The data of this database includes the tables tracks, artists, users, user interests, recommendations, user rates et cetera. For starting the tables of tracks and specialists, a C\# code was created to recover music data from social network last.fm. The information was gathered inside three weeks including 137685 tracks and 2125 artists after filtration of some invalid information in the gathered dataset. For both tables of tracks and artists by RESTful queries, the data was procured and accordingly, a XML stream of information containing of the details of tracks and artists was gotten. The exploration space was restricted to Persian music tracks and specialists in light of the fact that the users who were considered to work with the framework were Iranian individuals, in this way just those music tracks which were performed by Iranian artists and well known for the users, were gathered so they could have important action.

The demonstration of giving trust of the data, that the Confidentiality, Integrity and Availability (CIA) of the data are not damaged. For example, guaranteeing that information is not lost when basic issues emerge. These issues incorporate, however are not restricted to: regular debacles, PC/server glitch, physical burglary, or whatever other case where information has the capability of being lost. Since most data is put away on PCs in our advanced period, data confirmation is regularly managed by IT security authorities. A typical technique for giving data affirmation is to have an off-site reinforcement of the information in the event that one of the said issues emerges.

\section{EVALUATION AND RESULTS}

In this part of paper, the outcome of the web application as a case study for the suggested framework is validated. Therefore, it is possible to conclude that the framework has an appropriate efficiency and the results of the evaluation have been able to prove the previous assertions along with an improved framework rather than the previous established works with a novelty in its design.

\subsection{EXPERIMENTAL SETUP}

The users have worked with the web application (SARSIS) on the online platform. So the data needed for the evaluation section is located on the database server. To establish the experimental setup, first it was necessary to transfer the online data to the local machine as offline so 
that working with the desired data would be easy to use and more ready to handle.

Table 1 Statistics about the database of the web application

\begin{tabular}{|c|c|c|}
\hline $\begin{array}{c}\text { Table } \\
\text { name }\end{array}$ & Description & $\begin{array}{c}\text { Number } \\
\text { of rows }\end{array}$ \\
\hline Artists & Details of music artists & 2128 \\
\hline Tracks & Details of music tracks & 137685 \\
\hline Users & $\begin{array}{c}\text { Registered users, working on the web } \\
\text { application }\end{array}$ & 73 \\
\hline Interests & $\begin{array}{c}\text { Interesting music tracks for the users } \\
\text { by their activity }\end{array}$ & 1096 \\
\hline Recs & $\begin{array}{c}\text { Recommended music tracks by the } \\
\text { web application (Framework) }\end{array}$ & 6690 \\
\hline UserRates & $\begin{array}{c}\text { User rates about the recommendations } \\
\text { In the evaluation page by the users }\end{array}$ & 625 \\
\hline
\end{tabular}

For this purpose, a web page was developed using ASP.NET C\# that could do the appropriate data movement. After that, it was possible to analyze data easily.

List of experimental information comprising the tables of the prepared database is shown in Table 1 after collecting information from the users' activities on the website.

In this part of the paper, the gathered results of the mentioned case study of the framework are explained. First, the information is pointed about the users who have worked with SARSIS. The age classes of the users are shown in

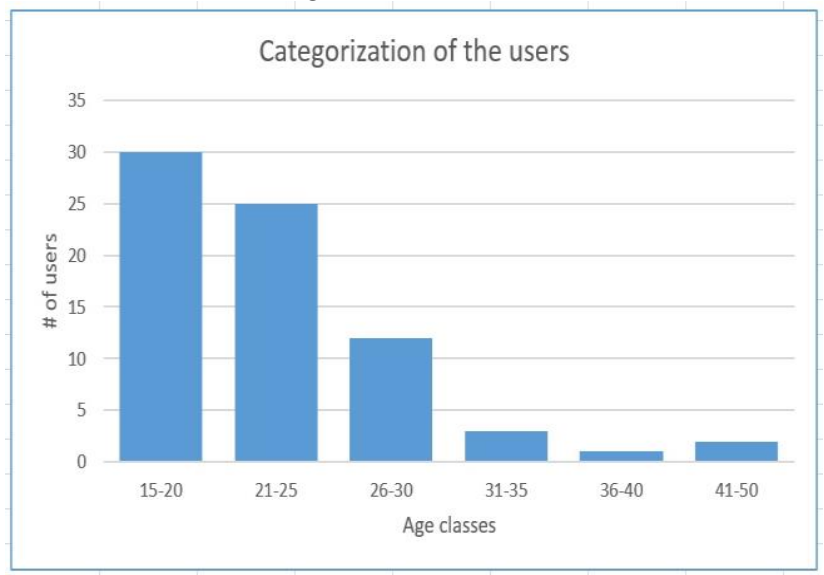

Figure 2.

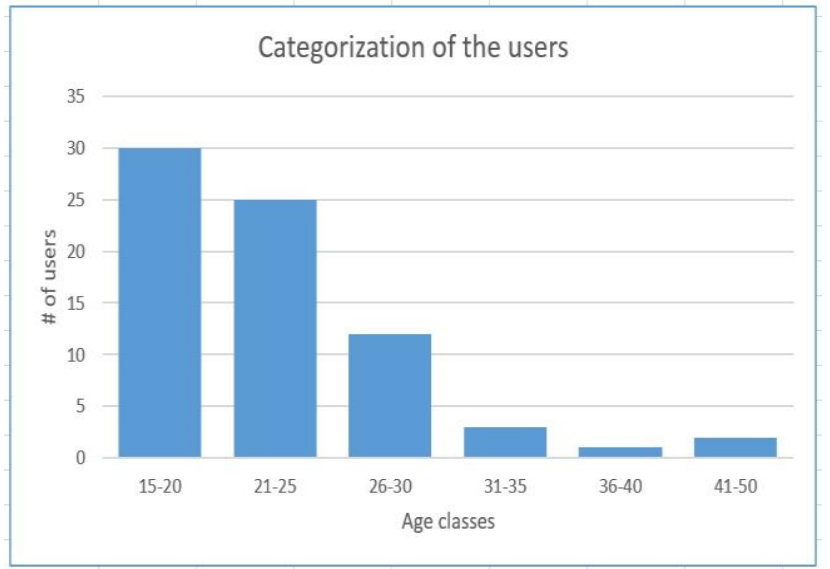

Figure 2 Categorization of the users based on age classes

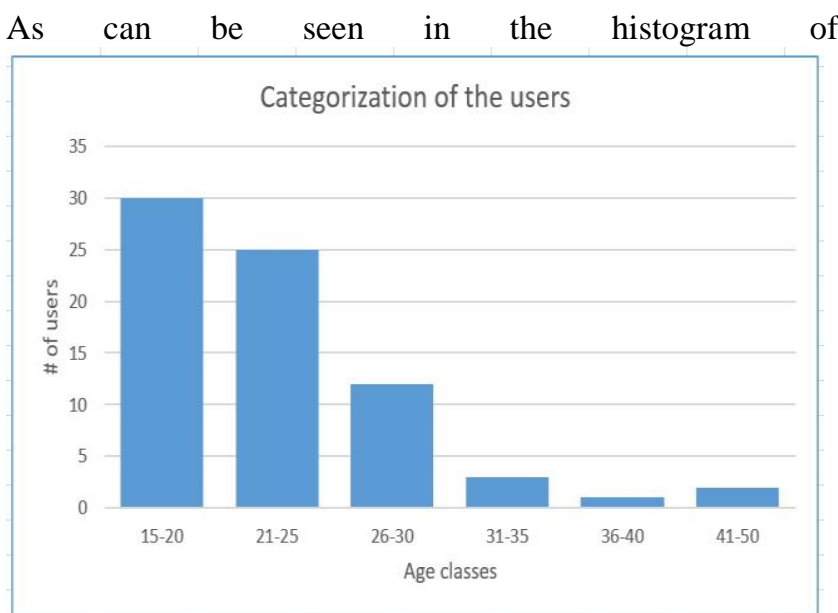

Figure 2, most of the users who participated in the research work are belong to the ages between 15 and 25 .

By analyzing the table UserRates and the number of liked music tracks which each user has submitted, using the Equation (8), it is possible to get the result using a SQL query which is executed in MS SQL Server Management Studio. The query shown in the Equation 8 depicts how the results can be prepared using a SQL query. The lower Mean Absolute Error (MAE), the more accurately the recommendation engine predicts user ratings. Root Mean Squared Error (RMSE), and Correlation are also used as statistical accuracy metric.

$$
M A E=\frac{\sum_{i=1}^{N}|p i-q i|}{\mathrm{N}}
$$


Equation 8 Calculation of MAE

The information of the outcome which is produced by the query of the Figure 3, is shown in

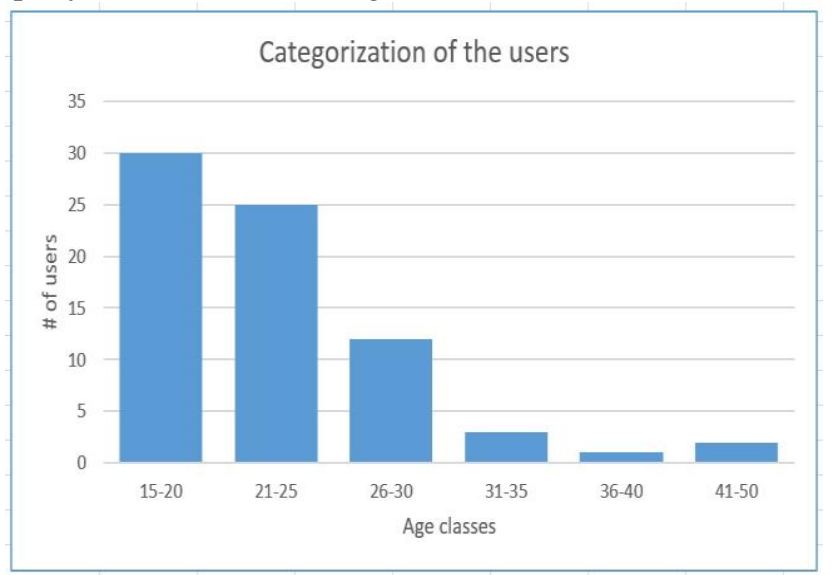

Figure 2 as a graph of calculated values. Furthermore, the calculated MAE for each user is depicted in Figure 4.
Figure 3 The SQL query

The maximum value, as the top of this graph is 1 . The horizontal axis presents the User IDs of the users and the vertical axis shows the values of MAE for each User ID. Another graph has been depicted the number of unliked recommended music tracks versus the number of recommended music tracks for each user which is shown as Figure 5. The overall MAE for the total users, or on the other word for the whole of the web application, can be estimated as Equation 9.

$$
\mathrm{MAE}=\frac{157}{625}=0.2512
$$

Equation 9 Calculation of total MAE for the whole of web application
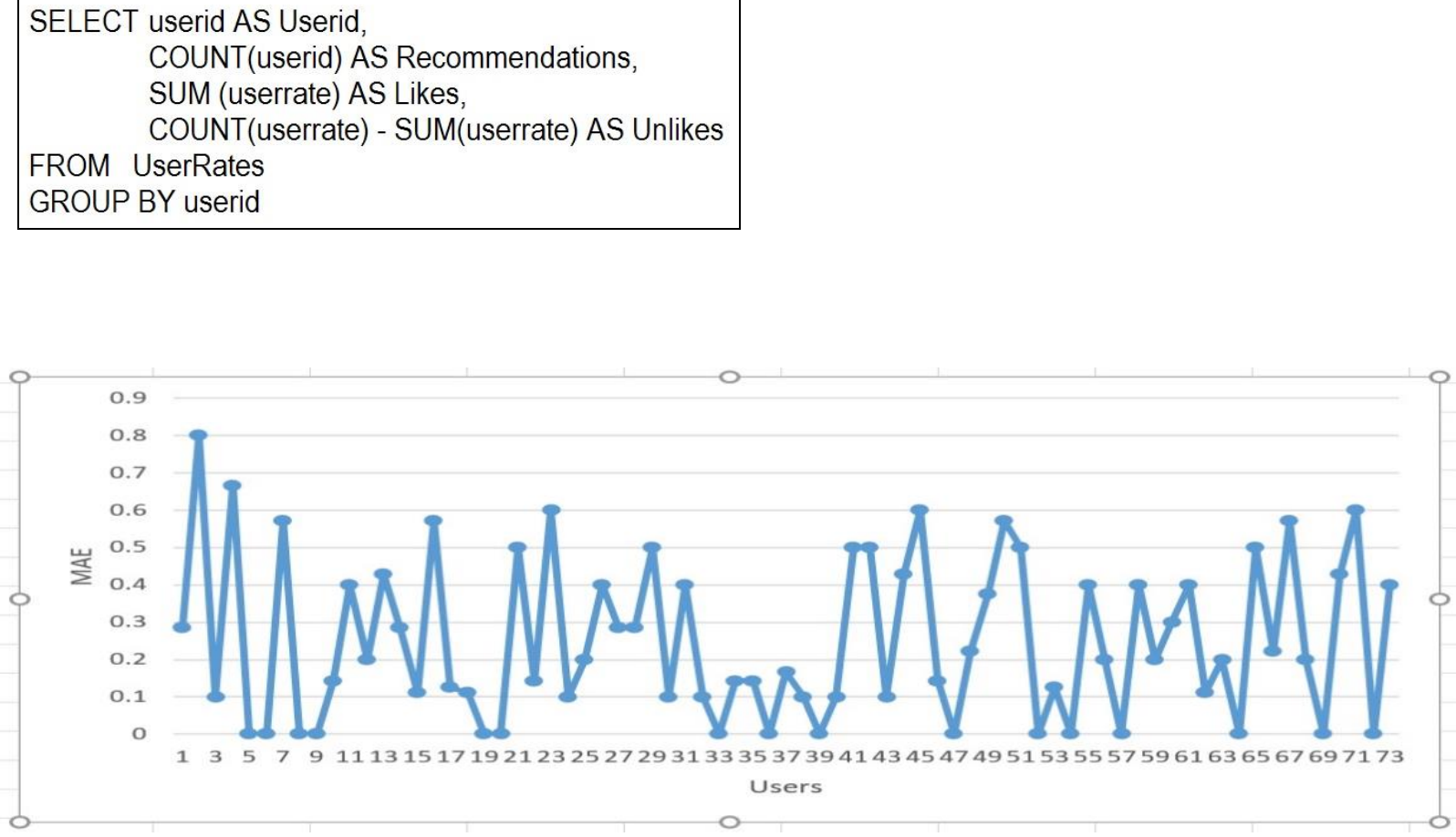

Figure 4 The calculated MAE for each user 


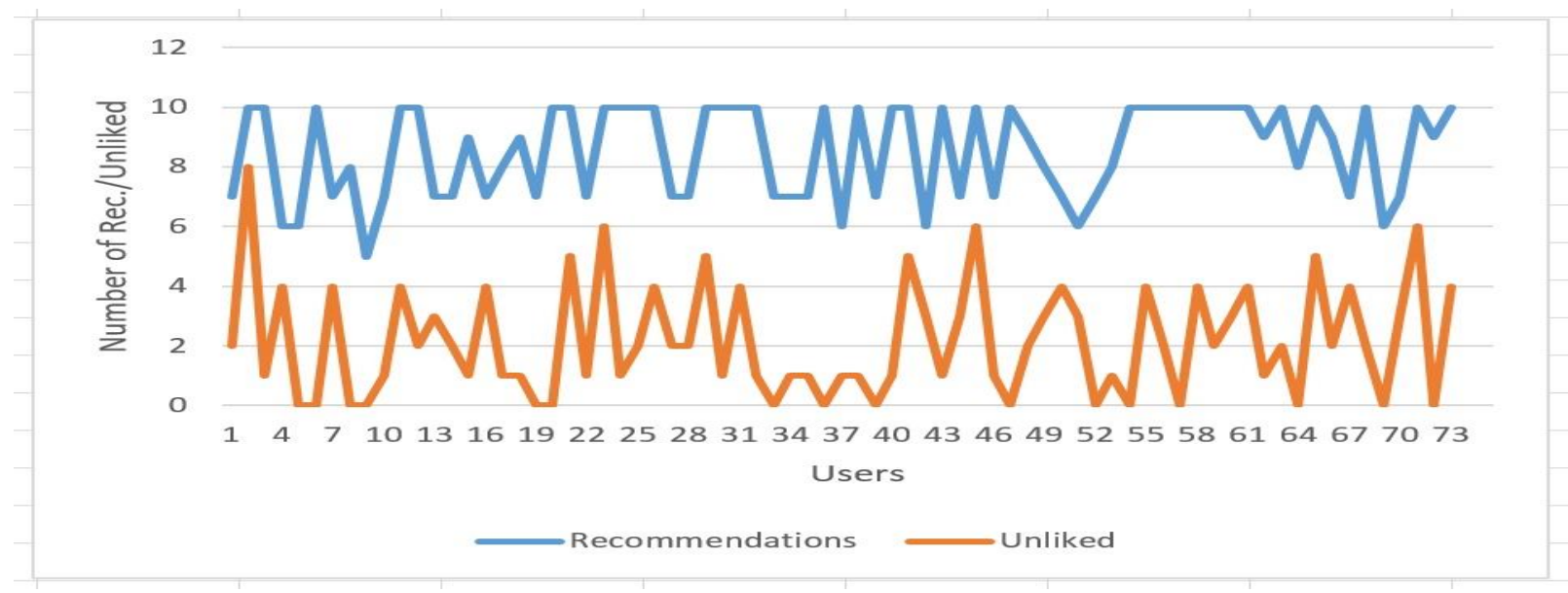

Figure 5 The numbers of unliked recommended music tracks versus the number of recommended music tracks for each user

They are shown in Figure 6 as the precision and recall curves separately next to each other and Figure 7 as a precision-recall curve. The average accuracy of the whole system as a representative of precisions that is estimated 0.7498 . The gathered results showed that the efficiency of the designed case study, as a sample for the framework, is adequate. For reaching this aim, an evaluation criterion as the Mean Absolute Error was used and calculated to prove the accuracy of the framework.

Although the value of MAE for whole of the web application was not very low but it was promising and acceptable. One reason about the value of MAE which is a little bit relatively high after interview with some of the users was that there is no possibility of finding and listening to a preview for some of the music tracks. As a result, they could not like the music track and as an outcome, the number of liked music tracks was reduced that influenced directly in estimating of MAE. So, if it was possible to select a more comprehensive website of music track resources, absolutely the value of MAE could be decreased accordingly. But the problem was that after doing an investigation and comparing many websites as the resources of music track which could be used for playing the previews, the best choice among distinguished websites was Spotify. Moreover, based on the Figure 6 and Figure 7, it is possible to see positive values for precision and recall for the users.

In total, the results and the gathered data from user rates, which reflects their feedback about the framework, proves that they are generally satisfied about the recommendations. So, based on this information we can conclude that if the framework can be implemented on a social network and use semantic methods to provide advertisements, the results will have enough efficiency for the business which consider this framework.

Here, the results of this research are compared with the other similar researches. As described in previous sections and pointing to these papers (9) (17) (18), it is possible to see the differences between our study and the other more similar studies. First the work (18) is referred whose application's name is SPAC. A comparison of accuracy between SARSIS and SPAC has been depicted in Figure 8. On average, SPAC showed a better accuracy rather than SARSIS. 


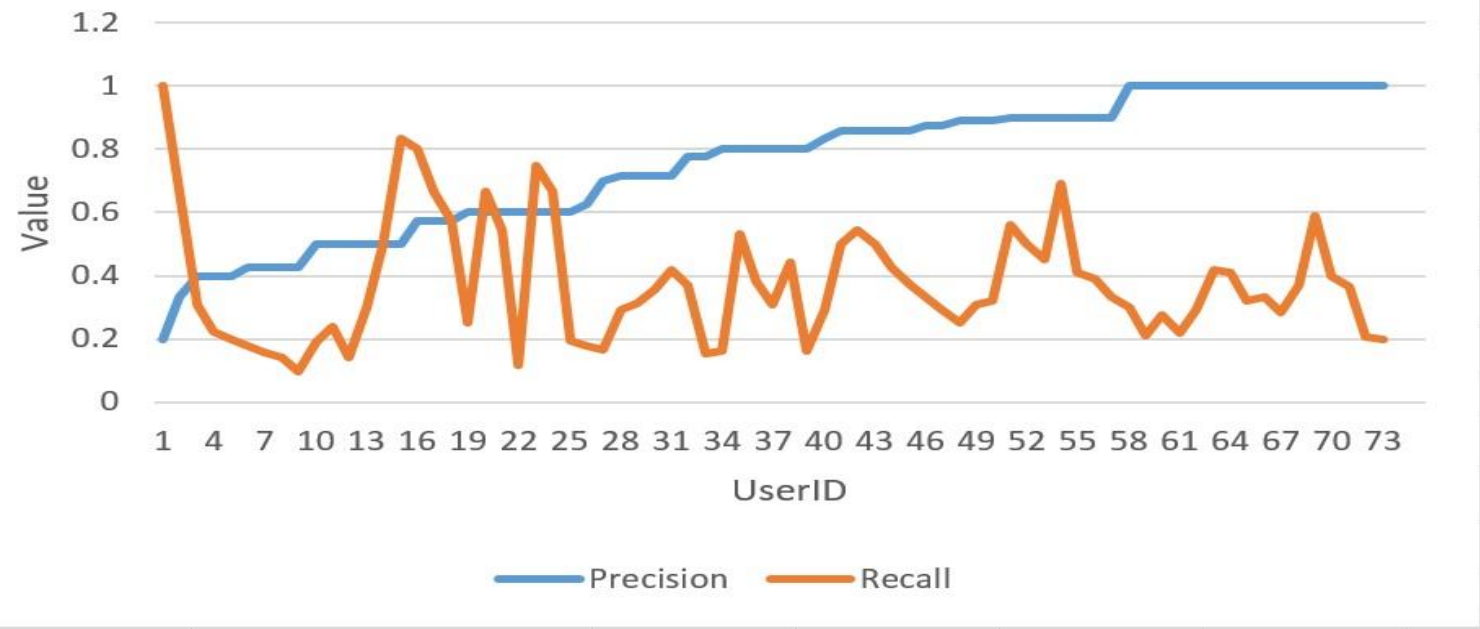

Figure 6 Precision and Recall curves

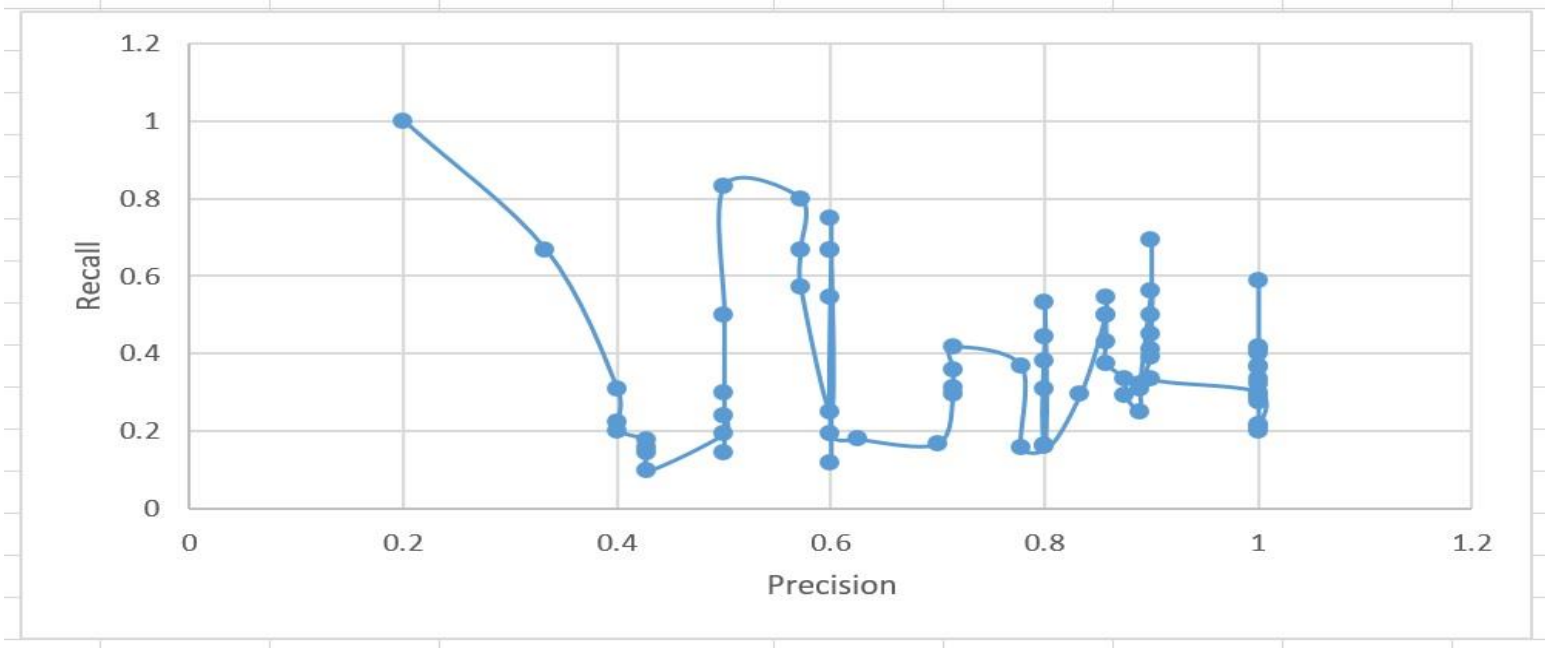

Figure 7 Precision-Recall Curve

Second, the study (9) is pointed. A graph including the MAE in SARSIS and this system has been shown in Figure 9. According to the shown graph, it can be concluded that SARSIS has a better efficiency with lower average MAE. Third, the study (17) is referred whose application's name is Friendbook. A comparison between Recall of SARSIS and Friendbook has been depicted in Figure 10.

According to this graph, SARSIS has a lower average recall with a higher average precision rather than Friendbook. So it can be concluded that SARSIS has a better efficiency than Friendbook.

Furthermore, there were some restrictions in this study which did not permit to do the research with more degree of quality. For example, among API functions of the social network, last.fm. as the case study of the social network, there was not a sign up method which could not be prepared as a platform for the users of web application to join the last.fm and have activity on its own interface. In this case, it could be possible to assess the efficiency of recommender system, which is used in last.fm. Therefore, it could not be provided more comprehensive comparison details between SARSIS and the recommender system of last.fm.

One difficulty in doing this research was that there was no suitable prepared dataset to be utilized in the web application. As a result, a customized dataset should be prepared based on the real data of a social network. Then, there was a try to collect data from selected social network, last.fm, including the important data as music tracks with 137685 records and artist people with 2125 records which was crawled from this social network by several REST queries and it took a complete three weeks of time by an intensive hard work. 


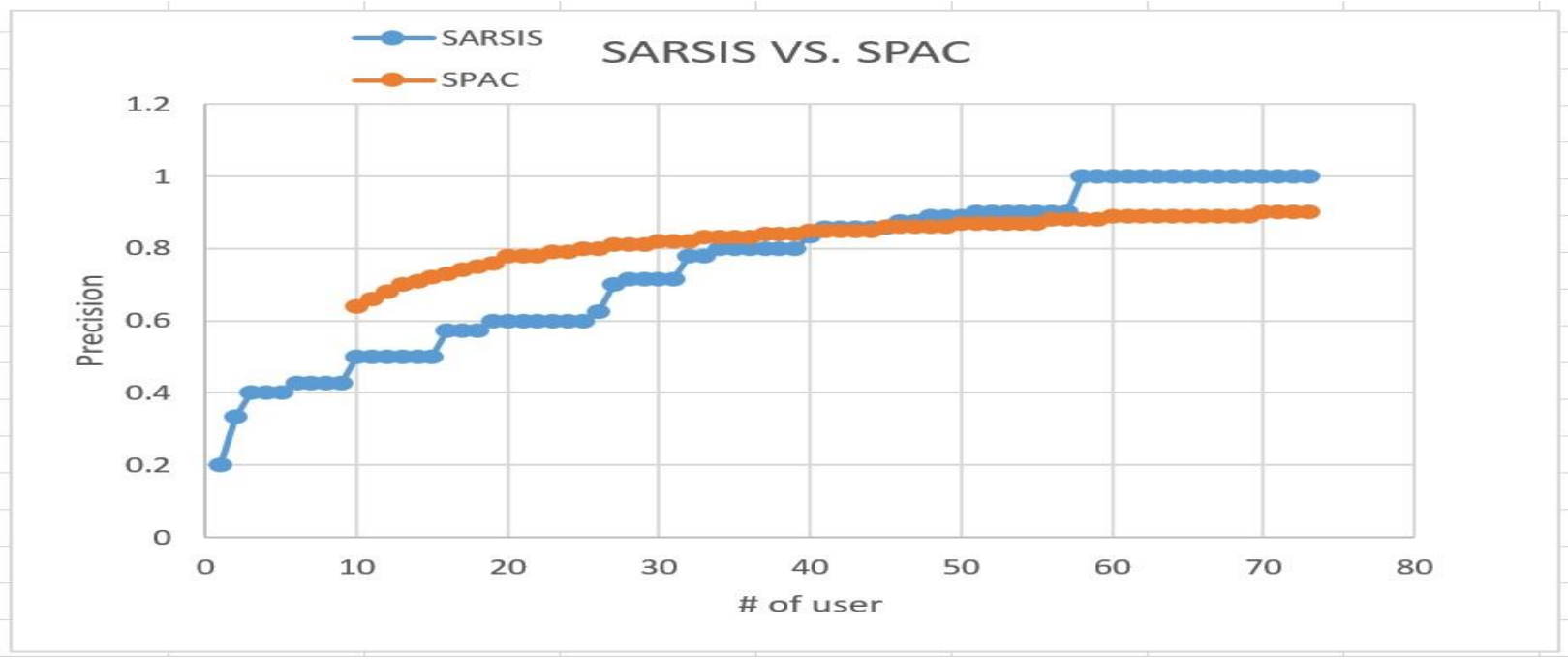

Figure 8 A comparison of accuracy between SARSIS and SPAC

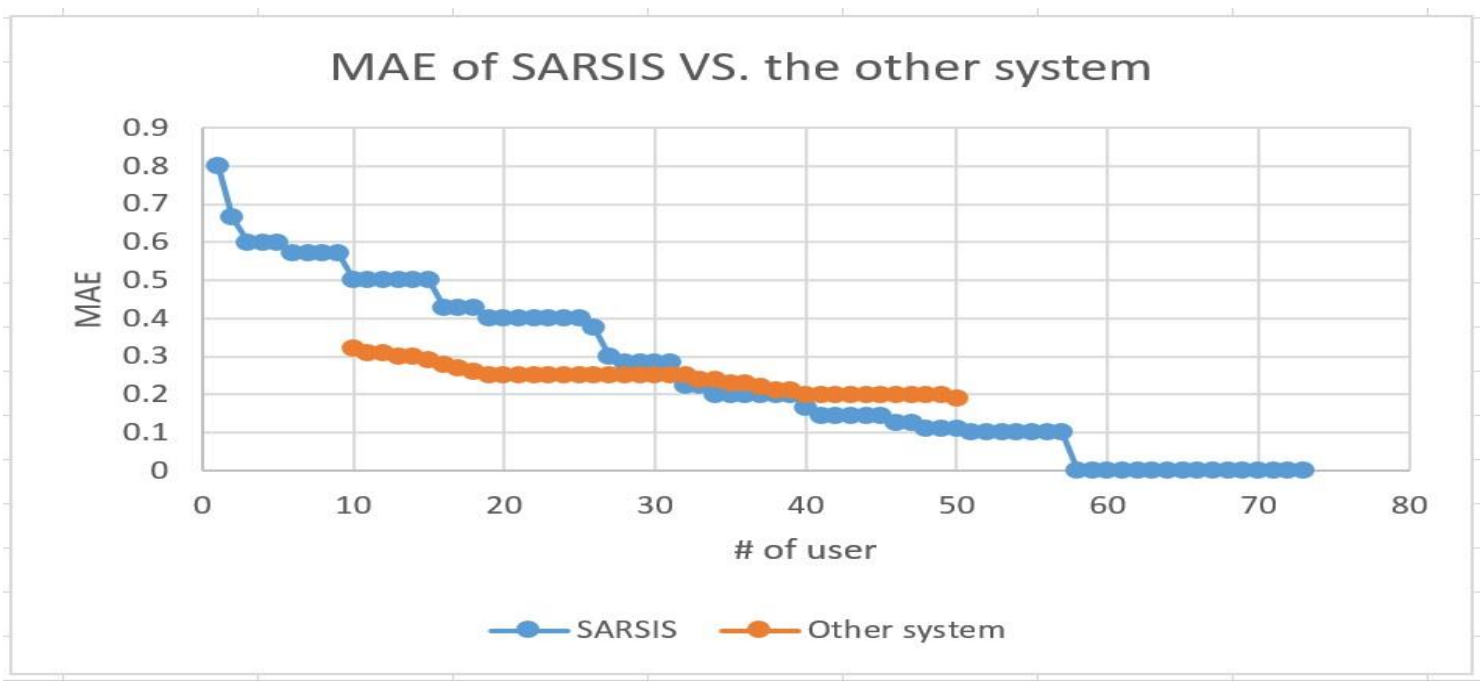

Figure 9 A comparison of MAE between SARSIS and the other system

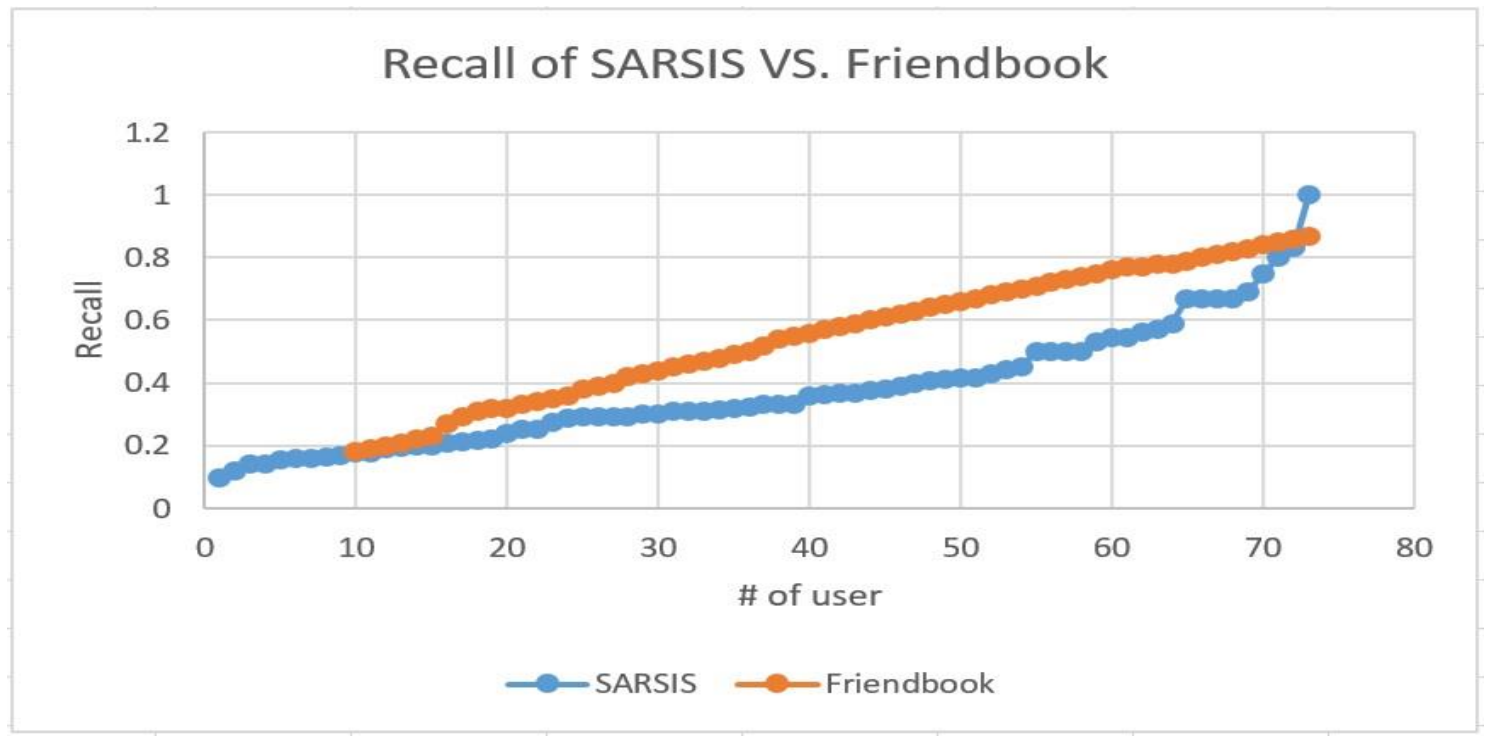

Figure 10 A comparison of Recall between SARSIS and Friendbook 


\section{CONCLUSION AND FUTURE WORKS}

In this paper, a semantically-enhanced advertisement recommender system for social networks has been described. The key strengths of the framework are summarized as follows: (1) an appropriate level of security has been considered, (2) using semantics improves recommendation precision and robustness, (3) a mathematical formulas allow estimating the recommender methods that are part of the hybrid framework, and (4) users' opinions are considered to continuously update the recommendations knowledge base so keeping it accurate and up-to-date.

A number of relevant issues are left for future work:

1- To define different levels of accessing or access control for the security part of the framework.

About the security of the framework, it is a good idea to develop its structure by enhancing some part of its security and enriching a multi factor authentication for the user entrance.

\section{REFERENCES}

[1] Li Y, Zhang D, Lan Z, Tan K. Context-Aware Advertisement Recommendation for High-Speed Social News Feeding. 2016;505-16.

[2] Pumphrey C. How do advertisers show me custom ads? [Internet]. 2012. Available from: http://computer.howstuffworks.com/advertiser-customads.htm

[3] Keating MG. Benefits of Targeted Advertisements: A Spotify Fail [Internet]. 2013. Available from: https://www.ereach.net/benefits-of-targetingadvertisements/

[4] Pleshette LA. Advantages and Disadvantages of Various Advertising Mediums [Internet]. 2013. Available from: http://www.powerhomebiz.com/marketingtips/advertising/advantages-and-disadvantagesadvertising-mediums.htm

[5] Wikipedia. Social media marketing [Internet]. 2016. Available

from: https://en.wikipedia.org/wiki/Social_media_marketing

[6] Breslin, John G.; Passant, Alexandre, and Decker S. The Social Semantic Web". Springer, 1st Ed. 2009;

[7] Wikipedia. Cold start [Internet]. 2016. Available from: https://en.wikipedia.org/wiki/Cold_start

[8] Zadeh PM, Moshkenani MS. Mining social network for semantic advertisement. Proc - 3rd Int Conf Converg Hybrid Inf Technol ICCIT 2008. 2008;1:611-8.

[9] Zaman N, Li J. Semantics-enhanced recommendation system for social healthcare. Proc - Int Conf Adv Inf Netw Appl AINA. 2014;765-70.

[10] Martín-Vicente MI, Gil-Solla A, Ramos-Cabrer M, Blanco-Fernández Y, Servia-Rodríguez S. Semanticsdriven recommendation of coupons through Digital TV: Exploiting synergies with social networks. Dig Tech Pap
2- To run the web application once again with more number of users to see the differences of outcomes and get better results.

In the future, if possible, it is preferred to run some times more to get a more volume of data by users' activities and after that do a comprehensive analysis on the collected data. As a result, the outcome will have more accuracy and consequently the users will be more satisfaction about the framework.

3- To find a more comprehensive resource of products for showing the details in the implementation section of the case studies.

4- If more social network matched to this framework can be emerged in the future, it is preferred to run and test the framework on more social networks. The social network should have flexible API with easy to use syntax for developing.

\section{ACKNOWLEDGEMENT}

This work has been partially supported by the Spanish MINECO Grant RACHEL TEC2013-47141-C4-4-R.

- IEEE Int Conf Consum Electron. 2012;564-5.

[11] Jo H-R, Park K-W, Kim J-I, Lee D-H. A Semantic Category Recommendation System Exploiting LDA Clustering Algorithm and Social Folksonomy. 2015 IEEE 39th Annu Comput Softw Appl Conf [Internet]. 2015;644-5. Available from: http://ieeexplore.ieee.org/lpdocs/epic03/wrapper.htm?ar number $=7273446$

[12] Chen L, Shao C, Zhu P. Social recommendation using quantified social tie strength. 2015 7th Int Conf Adv Comput Intell ICACI 2015. 2015;84-8.

[13] Oechslein O, Hess T. The value of a recommendation: The role of social ties in social recommender systems. Proc Annu Hawaii Int Conf Syst Sci. 2014;1864-73.

[14] Wang J, Liu Z, Zhao H. Group recommendation using topic identification in social networks. Proc - 2014 6th Int Conf Intell Human-Machine Syst Cybern IHMSC 2014. 2014;1:355-8.

[15] Hariri N, Mobasher B, Burke R. Using social tags to infer context in hybrid music recommendation. Proc twelfth Int Work Web Inf data Manag - WIDM '12 [Internet]. 2012;5. Available from: http://dl.acm.org/citation.cfm?doid=2389936.2389946

[16] Resnick P, Varian H. Recommender systems. Commun ACM [Internet]. 1997;1-21. Available from: http://dl.acm.org/citation.cfm?id=245121

[17] Wang Z, Liao J, Cao Q, Qi H, Wang Z. Friendbook: A semantic-based friend recommendation system for social networks. IEEE Trans Mob Comput. 2015;14(3):538-51.

[18] Li Q, Li J, Wang H, Ginjala A. Semantics-enhanced privacy recommendation for social networking sites. Proc 10th IEEE Int Conf Trust Secur Priv Comput Commun Trust 2011, 8th IEEE Int Conf Embed Softw Syst ICESS 2011, 6th Int Conf FCST 2011. 2011;22633. 